See discussions, stats, and author profiles for this publication at: https://www.researchgate.net/publication/303030865

\title{
Evaluation of rotated upwind schemes for contact discontinuity and strong shock
}

Article in Computers \& Fluids · August 2016

DOl: 10.1016/j.compfluid.2016.05.010

CITATIONS

14

4 authors, including:

Fan Zhang

KU Leuven

32 PUBLICATIONS 56 CITATIONS

SEE PROFILE

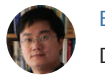

Dalian University of Technology

41 PUBLICATIONS 458 CITATIONS

SEE PROFILE

Some of the authors of this publication are also working on these related projects:

Project Embedded discontinuity-fitting method on unstructured dynamic grid View project

Project Spatial reconstructions of cell-centered FVM View project
READS

177

Jun Liu

Dalian University of Technology

123 PUBLICATIONS 202 CITATIONS

SEE PROFILE 


\title{
Evaluation of rotated upwind schemes for contact discontinuity and strong shock
}

\author{
Fan Zhang ${ }^{\mathrm{a}, \mathrm{c}, *}$, Jun Liu ${ }^{\mathrm{b}}$, Biaosong Chen ${ }^{\mathrm{a}, \mathrm{c}}$, Wanxie Zhong ${ }^{\mathrm{a}, \mathrm{c}}$ \\ ${ }^{a}$ Department of Engineering Mechanics, Dalian University of Technology, Dalian, China \\ ${ }^{b}$ School of Aeronautics and Astronautics, Dalian University of Technology, Dalian, China \\ ${ }^{c}$ State Key Laboratory of Structural Analysis for Industrial Equipment, Dalian University of Technology, \\ Dalian, China
}

\begin{abstract}
Rotated upwind schemes have been evaluated as carbuncle-free shock-capturing methods. In order to reveal the relation between the rotated upwind-differencing direction and the performance of rotated upwind schemes, theoretical and numerical investigations are made. Three commonly used upwind schemes, including Roe scheme, $\mathrm{AUSM}^{+}$scheme and Van Leer scheme are investigated. According to the theoretical analysis, the dissipative terms of each upwind scheme are changed by the rotation procedure. Especially, the dissipative density term in the numerical flux function of rotated upwind schemes introduces extra dissipation. The numerical cases include simulations of laminar boundary layer, steady twin vortices and strong shock. The numerical result$\mathrm{s}$ show that the carbuncle phenomenon can be eliminated by rotated upwind schemes, and the contact resolving capability of rotated upwind schemes is deteriorated by the rotation procedure. Monotonicity of the dissipation with rotation angle is also shown in the numerical results. Therefore, a novel rotation strategy that defines the rotation angle by a pressure weight function is introduced for tuning the dissipative effects adaptively. This strategy shows not only carbuncle-free result in hypersonic inviscid flow simulations, but also accurate results in viscous flow simulations.
\end{abstract}

Keywords: rotated upwind scheme; carbuncle phenomenon; shock-capturing; dissipative density term; contact discontinuity; rotation strategy

\footnotetext{
${ }^{*}$ Corresponding author.

Email address: a04051127@mail.dlut.edu.cn (Fan Zhang)
} 


\section{Introduction}

Hypersonic flow simulations are very challenging due to shock instabilities, such as carbuncle phenomenon [1], odd-even decoupling [2] and so on. In general, shock instabilities are more prominent and complicate in multidimensional simulations [3, 4].

5 Researches have shown that the shock instabilities are closely related to the resolution of contact discontinuity and shear wave. Liou [5] analysed the mechanism of numerical diffusivity of several numerical flux schemes. He suggested that the dissipative pressure term would be a key factor for shock stability. Pandolfi and D'Ambrosio [6] suggested that the carbuncle phenomenon affects flux-difference-splitting (FDS) schemes that explicitly take into account the presence of contact surface. In fact, for an upwind scheme, research suggested that strict stability and exact resolution of contact discontinuity are not compatible [7]. Therefore, devise a combined upwind scheme [8] or a tunable dissipation would be a practical strategy for acquiring shock stability and contact resolution at the same time. Quirk [2] suggested that using combined fluxes, then a dissipative flux can be used in the shock regions. In contrast, in stead of a different upwind scheme, second-order artificial dissipation could be directly added on a flux-vector-splitting (FVS) method [9] in the shock regions [10,11].

Because shock instabilities are multidimensional numerical phenomena, some researches have taken the multidimensional effects as a critical factor for the construction of numerical fluxes. The rotated Riemann solver, as one type of the multidimensional upwind methods [12], was showing an alterable dissipation that can be adaptively activated to suppress shock instability [13]. As one of the pioneer works, in order to lessen the dependence between numerical solution and grid orientation, Levy et al [12] examined a "rotated Riemann solver" by exploiting the multidimensional nature of the governing equations. Several physical meaningful directions were used as the direction for upwind differencing. In Levy's work, the approximate Riemann solver of Roe [14] was implemented to calculate fluxes in both the upwind direction and its perpendicular direction, thus the rotated scheme requires additional computation resources over grid-aligned schemes. Besides the efficiency issue, the choice of the upwind differencing direction and the combination of upwind schemes were investigated by subsequent 
researches.

Ren [13] presented a rotated Roe scheme which is free from shock instabilities. In Ren's work, the upwind-differencing direction was defined as the direction of the velocity-difference vector across each grid interface. The dissipation properties of the rotated flux function was theoretically analysed. Different from Ren's work, Nishikawa and Kitamura [15] combined Roe scheme and HLL scheme [16] for eliminating shock instabilities. Thereinto, a fewer-wave solver (HLL) was automatically applied in the direction normal to the shocks, and then shock instabilities will be suppressed. A fullwave solver (Roe) was applied across shear layers to avoid an excessive amount of dissipation. In Nishikawa and Kitamura's work, simulations of supersonic jet and flat plate boundary layer were conducted for the demonstration of the shear layers resolving capability. Zha et al [17] used ECUSP scheme $[18,19]$ instead of Roe scheme for the flux solution in the direction parallel to the shocks. Huang et al [20] managed to resolve the problem of the numerical shock instabilities in HLLC solver [21], and one of their method was the rotated HLLC scheme.

After all these works, problems still exist. For example, flat plate boundary layer simulation has been commonly used for evaluating the contact and shear layer resolving capability of upwind schemes. However, rotated upwind schemes usually become a grid-aligned scheme (rotate to the face normal direction) in the flat plate boundary layer simulation. The problem is, even in a shock-free simulation, velocity difference vector direction (or the other specific upwind-differencing directions) is not always parallel or perpendicular to the normal direction of interface. Furthermore, in order to acquire a full convergence, the upwind-differencing direction vector was suggested to be freezed after an order of magnitude reduction of the residuals [15]. In other words, the actual upwind-differencing direction does not strictly coincide with the suggested flow features. Therefore, it is necessary to investigate the relation between the dissipation property and rotation angle of rotated upwind schemes specifically.

In this paper, rotated upwind schemes are investigated based on former researches. The numerical mass flux, which was investigated by Liou [5] and well utilized by Kim 60 et al [22], is used for analysing the performance of rotated schemes. The presented paper is organized as follows. In the next section, a finite volume formulation of the 
governing equations is given. In section 3, basic concepts of rotated upwind schemes are presented, as well as the basic rotation strategy applied in this paper. Theoretical analyses are introduced in section 4 . Numerical experiments are presented in section 5 to further clarify the conclusions in section 4 . Then the new rotation strategy could be proposed straightforwardly, as presented in section 6 . Finally, the paper ends with the conclusions.

\section{Finite Volume Discretization}

Consider the two-dimensional compressible Navier-Stokes equations,

$$
\frac{\partial \mathbf{U}}{\partial t}+\frac{\partial\left(\mathbf{F}_{c}-\mathbf{F}_{v}\right)}{\partial x}+\frac{\partial\left(\mathbf{G}_{c}-\mathbf{G}_{v}\right)}{\partial y}=\mathbf{0} .
$$

The viscous fluxes $\mathbf{F}_{v}$ and $\mathbf{G}_{v}$ are solved by a central scheme for unstructured grid [23]. The viscous fluxes, of which the solution method is not investigated in this work, will be omitted for simplicity. Then the simplified equations become the Euler equations. The conservative variables and convective fluxes are

$$
\mathbf{U}=\left[\begin{array}{c}
\rho \\
\rho u \\
\rho v \\
\rho E
\end{array}\right], \mathbf{F}_{c}=\left[\begin{array}{c}
\rho u \\
\rho u^{2}+p \\
\rho u v \\
\rho u H
\end{array}\right], \mathbf{G}_{c}=\left[\begin{array}{c}
\rho v \\
\rho u v \\
\rho v^{2}+p \\
\rho v H
\end{array}\right],
$$

where $\rho$ is the density, $u$ and $v$ are the velocity components in the $x$ and $y$ directions respectively, and $p$ is the static pressure. The specific total energy $E$ and enthalpy $H$ are given by

$$
\begin{aligned}
& E=\frac{1}{\gamma-1} \frac{p}{\rho}+\frac{1}{2}\left(u^{2}+v^{2}\right), \\
& H=\frac{\gamma}{\gamma-1} \frac{p}{\rho}+\frac{1}{2}\left(u^{2}+v^{2}\right),
\end{aligned}
$$

where $\gamma=1.4$ for calorically perfect gas. Consider the cell-centered finite volume discretization, the computation domain is discretized to a set $\{I\}$ of quadrilateral and/or triangular cells. For each cell $i \in\{I\}$, have a set of faces $\left\{K_{i}\right\}$. Each face $k \in\left\{K_{i}\right\} \cap$ $\left\{K_{j}\right\}$ connects two neighbouring cells $i$ and $j$. The cell-residual $\operatorname{Res}_{i}$ is defined as a 
numerical approximation of the integral over the cell of the spatial operator divided by the cell area $S_{i}$, that is

$$
\boldsymbol{R e s}_{i}=-\sum_{k \in\left\{K_{i}\right\}} \boldsymbol{\Phi}_{k}\left(\mathbf{n}_{k}\right) \Delta s_{k} / S_{i},
$$

where the $\Delta s_{k}$ is the length of cell face $k \in\left\{K_{i}\right\}, \mathbf{n}_{k}$ is the unit vector outward normal to that face, and $\boldsymbol{\Phi}_{k}\left(\mathbf{n}_{k}\right)$ is the numerical flux, which is a numerical approximation to $\left[\mathbf{F}_{c}, \mathbf{G}_{c}\right] \cdot \mathbf{n}_{k}$. The cell-averaged value $\overline{\mathbf{U}}_{i}$ is then evolved by the residual as

$$
\frac{\partial \overline{\mathbf{U}}_{i}}{\partial t}=\operatorname{Res}_{i}
$$

which is integrated in time by the Forward-Euler method, Runge-Kutta method or LUSGS method.

The numerical flux $\boldsymbol{\Phi}_{k}\left(\mathbf{n}_{k}\right)$ is evaluated by using the left and right states of the interface. If the cell-averaged values are taken as the left and right states, the accuracy of the numerical method will be first-order in space. In order to achieve second-order accuracy, Gauss-Green method [24] is applied to calculate the cell gradient $\nabla \overline{\mathbf{U}}_{i}$. Therefore, the left and right states of face $k$ are

$$
\begin{aligned}
& \overline{\mathbf{U}}_{k}^{L}=\overline{\mathbf{U}}_{i}+\phi_{i} \nabla \overline{\mathbf{U}}_{i} \cdot\left(\mathbf{x}_{k}-\mathbf{x}_{i}\right), \\
& \overline{\mathbf{U}}_{k}^{R}=\overline{\mathbf{U}}_{j}+\phi_{j} \nabla \overline{\mathbf{U}}_{j} \cdot\left(\mathbf{x}_{k}-\mathbf{x}_{j}\right),
\end{aligned}
$$

where $i, j \in\{I\}$, and $k \in\left\{K_{i}\right\} \cap\left\{K_{j}\right\}$. In the following numerical investigations, the slope limiter of Venkatakrishnan [25] is used for the calculation of $\phi$ in second-order spatial accuracy supersonic flow simulations. Otherwise, the limitation value $\phi$ is simply set as 1 .

As mentioned by Ren [13], the rotated methods that use the same set of face data in every direction are efficient and can be easily extended to higher order of accuracy by using spatial interpolations or reconstructions. In this paper, the rotated schemes would be extended to second-order accuracy by the above formula, and thus the limiter can also be replaced by the other commonly used methods.

\section{Basics of rotated upwind schemes}

Reconsider Eq.1 in a numerical discretized form. The numerical flux $\boldsymbol{\Phi}_{k}\left(\mathbf{n}_{k}\right)$ across each face $k$ can be determined by solving the one-dimensional Euler equations in the 
direction of vector $\mathbf{n}_{k}$. The equations are

$$
\frac{\partial \mathbf{U}}{\partial t}+\frac{\partial \boldsymbol{\Phi}_{k}}{\partial \mathbf{n}_{k}}=\mathbf{0} .
$$

In what follows, the subscript $k$ is omitted for simplicity.

The rotated upwind schemes are based on the decomposition of direction $\mathbf{n}$ into two orthogonal directions $\mathbf{n}_{1}$ and $\mathbf{n}_{2}$,

$$
\mathbf{n}=C_{1} \mathbf{n}_{1}+C_{2} \mathbf{n}_{2}, \mathbf{n}_{1} \cdot \mathbf{n}_{2}=0,
$$

where $\mathbf{n}_{1}$ and $\mathbf{n}_{2}$ are unit vector, $C_{1}=\mathbf{n}_{1} \cdot \mathbf{n}$ and $C_{2}=\mathbf{n}_{2} \cdot \mathbf{n}$. In order to keep the same left and right states in both directions, $\mathbf{n}_{1}$ and $\mathbf{n}_{2}$ are chosen to make $C_{1} \geqslant 0$ and $C_{2} \geqslant 0$. Therefore, the numerical flux is then decomposed into the following form,

$$
\boldsymbol{\Phi}(\mathbf{n})=C_{1} \boldsymbol{\Phi}\left(\mathbf{n}_{1}\right)+C_{2} \boldsymbol{\Phi}\left(\mathbf{n}_{2}\right) .
$$

The decomposition of $\mathbf{n}$ into two vector $\mathbf{n}_{1}$ and $\mathbf{n}_{2}$ is purely a geometrical procedure. The physical features of flow field could be taken into account to determine the choices of $\mathbf{n}_{1}$ and $\mathbf{n}_{2}$. Usually, the vector $\mathbf{n}_{1}$ stands for some physically meaningful direction. For example, Ren [13] suggested that it would be effective and robust for Roe solver to align $\mathbf{n}_{1}$ with the velocity-difference vector between two adjacent cells,

$$
\mathbf{n}_{\mathbf{1}}=\left\{\begin{array}{cc}
\mathbf{n}, & \text { if }\|\Delta \mathbf{V}\| \leqslant \varepsilon \\
\frac{\Delta \mathbf{V}}{\|\Delta \mathbf{V}\|}, & \text { otherwise }
\end{array}\right.
$$

where $\Delta \mathbf{V}$ is the velocity-difference vector, $\varepsilon$ is a predesigned small threshold, and then $\mathbf{n}_{2}$ is given as

$$
\mathbf{n}_{2}=\frac{\left(\mathbf{n}_{1} \times \mathbf{n}\right) \times \mathbf{n}_{1}}{\left\|\left(\mathbf{n}_{1} \times \mathbf{n}\right) \times \mathbf{n}_{1}\right\|} .
$$

However, in this paper, the upwind differencing direction is defined in a different way. The decomposition of $\mathbf{n}$ is defined as

$$
\begin{gathered}
\mathbf{n}_{1}=\mathbf{T}_{1} \mathbf{n}, \mathbf{n}_{2}=\mathbf{T}_{2} \mathbf{n} \\
\mathbf{T}_{1}=\left[\begin{array}{cc}
\cos (\alpha) & \sin (\alpha) \\
-\sin (\alpha) & \cos (\alpha)
\end{array}\right], \mathbf{T}_{2}=\left[\begin{array}{cc}
\cos (\alpha-\pi / 2) & \sin (\alpha-\pi / 2) \\
-\sin (\alpha-\pi / 2) & \cos (\alpha-\pi / 2)
\end{array}\right],
\end{gathered}
$$


where $\alpha$ is the rotation angle at each interface. Therefore, the rotation could be controlled by defining the angle, and the effects of different rotation angles could be more observable. The detail of the designation of rotation angle will be introduced in section 856.

\section{Analyses}

The relation between the numerical mass flux and the shock stability or contact resolving capability was investigated theoretically and numerically by Liou [5]. Liou suggested that the existed pressure contribution to numerical mass fluxes will be the root for causing shock instabilities. Somewhat differently, Pandolfi and D'Ambrosio [6] suggested that the carbuncle phenomenon should be connected to those solutions of the Riemann problem that explicitly take into account the contact surface. Based on Liou's theorem, Kim et al [22] analysed and improved Roe scheme. Most importantly for the presented work, Kim et al proved that the perturbation introduced by the pressure term in the numerical mass flux function could be damped by a proper dissipative density term. So far, although debates and investigations are still carrying on the mechanism of shock instability, the method of Liou has proved to be helpful for analysing and improving the performance of upwind schemes.

An investigation on the dissipative terms of rotated upwind schemes is briefly performed in this section. The analysis method of Liou and Kim et al is adopted here. Following paragraphs first introduce the fluxes of Roe scheme, $\mathrm{AUSM}^{+}$(Advection Upwind Splitting Method) scheme [26] and Van Leer scheme [27]. Noteworthily, the Roe scheme was deemed as a carbuncle-prone scheme, the $\mathrm{AUSM}^{+}$scheme was considered as a light-carbuncle-prone scheme and the Van Leer scheme has been evaluated as a carbuncle-free scheme [6]. Subsequently, the flux function of each rotated upwind scheme is investigated by comparing with its grid-aligned counterpart. 


\subsection{Numerical mass fluxes of grid-aligned upwind schemes}

Based on the grid-aligned formulation, the numerical flux in Eq. 8 can be written as a split form $[5,22]$. Thereinto, the numerical mass flux is rewritten as

$$
\boldsymbol{\Phi}_{1 / 2}^{(\rho)}=\frac{1}{2}\left[\left(\rho V_{n}\right)_{L}+\left(\rho V_{n}\right)_{R}\right]-\frac{1}{2}\left[D^{(\rho)} \Delta \rho+D^{\left(V_{n}\right)} \Delta V_{n}+D^{(p)} \Delta p\right],
$$

where $V_{n}=[u, v] \cdot \mathbf{n}, D^{(\rho)}, D^{\left(V_{n}\right)}$ and $D^{(p)}$ are functions of mean quantities $\overline{\mathbf{U}}\left(\mathbf{U}_{L}, \mathbf{U}_{R}\right)$, and the difference operator is $\Delta()=(\bullet)_{R}-(\bullet)_{L}$. Because of their effects on the performance of upwind schemes, $D^{(\rho)}$ and $D^{(p)}$ will be investigated in the following paragraphs. The flux function of each grid-aligned scheme will be shown as follows.

For Roe scheme, the numerical flux could be written as [26]

$$
\begin{gathered}
\boldsymbol{\Phi}_{1 / 2}=\frac{1}{2}\left[\left(V_{n} \boldsymbol{\Phi}\right)_{L}+\left(V_{n} \boldsymbol{\Phi}\right)_{R}-|\hat{\boldsymbol{A}}| \Delta \boldsymbol{U}+\boldsymbol{P}_{L}+\boldsymbol{P}_{R}\right], \\
\boldsymbol{\Phi}_{L, R}=\left[\begin{array}{c}
\rho \\
\rho u \\
\rho v \\
\rho H
\end{array}\right]_{L, R}, \boldsymbol{P}_{L, R}=\left[\begin{array}{c}
0 \\
n_{x} p \\
n_{y} p \\
0
\end{array}\right]_{L, R}, \\
|\hat{\boldsymbol{A}}| \Delta \boldsymbol{U}=|\Delta \hat{\boldsymbol{\Phi}}|_{1}+|\Delta \hat{\boldsymbol{\Phi}}|_{2}+|\Delta \hat{\boldsymbol{\Phi}}|_{3}+|\Delta \hat{\boldsymbol{\Phi}}|_{4} .
\end{gathered}
$$

The over hat in the formulas means the variables are Roe's averaged approximations. In Eq.17, the terms in the RHS are represented as follows,

$$
\begin{aligned}
& |\Delta \hat{\mathbf{\Phi}}|_{1}=\left|\hat{V}_{n}\right|\left(\Delta \rho-\frac{\Delta p}{\hat{a}^{2}}\right)\left[\begin{array}{c}
1 \\
\hat{u} \\
\hat{v} \\
\frac{\hat{u}^{2}+\hat{v}^{2}}{2}
\end{array}\right],|\Delta \hat{\boldsymbol{\Phi}}|_{2}=\left|\hat{V}_{n}\right| \hat{\rho}\left(n_{y} \Delta u-n_{x} \Delta v\right)\left[\begin{array}{c}
0 \\
n_{y} \\
-n_{x} \\
\hat{u} n_{y}-\hat{v} n_{x}
\end{array}\right], \\
& |\Delta \hat{\mathbf{\Phi}}|_{3,4}=\left|\hat{V}_{n} \pm \hat{a}\right|\left(\frac{\Delta p \pm \hat{\rho} \hat{a} \Delta V_{n}}{2 \hat{a}^{2}}\right)\left[\begin{array}{c}
1 \\
\hat{u} \pm n_{x} \hat{a} \\
\hat{v} \pm n_{y} \hat{a} \\
\hat{H} \pm \hat{a} \hat{V}_{n}
\end{array}\right] .
\end{aligned}
$$


Therefore, the dissipative terms in the numerical mass flux of Roe scheme are [5, 22]

$$
\begin{aligned}
& D^{(\rho)}=\left|\lambda_{2}\right|, \\
& D^{(p)}=\left(\left|\lambda_{1}\right|+\left|\lambda_{3}\right|-2\left|\lambda_{2}\right|\right) / 2 \hat{a}^{2},
\end{aligned}
$$

where $\left\{\lambda_{k}, k=1,2,3\right\}=\left\{\hat{V}_{n}-\hat{a}, \hat{V}_{n}, \hat{V}_{n}+\hat{a}\right\}, \hat{a}$ is the speed of sound.

For $\mathrm{AUSM}^{+}$scheme, the numerical flux is written as [26]

$$
\boldsymbol{\Phi}_{1 / 2}=\frac{1}{2} a_{1 / 2}\left[M_{1 / 2}\left(\boldsymbol{\Phi}_{L}+\boldsymbol{\Phi}_{R}\right)-\left|M_{1 / 2}\right|\left(\boldsymbol{\Phi}_{R}-\boldsymbol{\Phi}_{L}\right)\right]+\boldsymbol{P}_{1 / 2},
$$

where the subscript $1 / 2$ is not an average of left and right states, but rather a weighted function. Therefore, the dissipative terms of $\mathrm{AUSM}^{+}$scheme are [5]

$$
\begin{aligned}
& D^{(\rho)}=\left|a_{1 / 2} M_{1 / 2}\right|=\left|V_{1 / 2}\right|, \\
& D^{(p)}=0,
\end{aligned}
$$

where $M_{1 / 2}=\mathscr{M}_{(\beta)}^{+}\left(M_{L}\right)+\mathscr{M}_{(\beta)}^{-}\left(M_{R}\right)$ is the surface mach number, left and right Mach numbers are defined as $M_{L, R}=\left(V_{n}\right)_{L, R} / a_{1 / 2}, a_{1 / 2}$ is defined by Liou [26].

The Van Leer scheme, which is a typical FVS scheme, could be written as

$$
\boldsymbol{\Phi}_{1 / 2}=\boldsymbol{\Phi}_{L}+\boldsymbol{\Phi}_{R}
$$

where $\boldsymbol{\Phi}_{L, R}$ were defined by van Leer [27] and different from Eq.16. While $|M|<1$, the mass flux of Van Leer scheme is

$$
\boldsymbol{\Phi}_{L, R}^{(\rho)}= \pm\left[\frac{\rho a}{4}(M \pm 1)^{2}\right]_{L, R} .
$$

Therefore, according to Eq.14, the dissipative terms of Van Leer scheme are written as [5]

$$
\begin{aligned}
& D^{(\rho)}=a_{L} \mathscr{M}_{(0)}^{+}\left(M_{L}\right)-a_{R} \mathscr{M}_{(0)}^{-}\left(M_{R}\right), \\
& D^{(p)}=0,
\end{aligned}
$$

where $M_{L, R}=\left(V_{n}\right)_{L, R} / a_{L, R}$.

The split functions in Eq.21 and Eq.24 are defined here to facilitate the discussion:

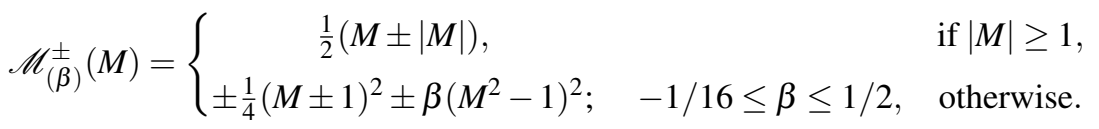

The parameter $\beta$ is set to meet the requirements on monotonicity, consistency and continuously differentiability of the split function [26]. For the $\mathrm{AUSM}^{+}$scheme, $\beta$ is usually set as $1 / 8$. For the Van Leer scheme, $\beta$ is always equal to zero. 


\subsection{Numerical mass fluxes of rotated upwind schemes}

The terms $D^{(\rho)}$ and $D^{(p)}$ have proved to be critical factors for the performance of upwind schemes. Thereinto, the exact solution for a contact discontinuity moving with speed $u_{c}$ requires that [5]

$$
D^{(\rho)}=\left|u_{c}\right|, \quad \text { as }(u, p)_{L}=(u, p)_{R}=\left(u_{c}, p\right) \text { and } \rho_{L} \neq \rho_{R} .
$$

following results are presented. It should be noticed again that the $C_{1}$ and $C_{2}$ in the following equations will not be smaller than zero by the proper choices of $\mathbf{n}_{1}$ and $\mathbf{n}_{2}$.

For the rotated Roe scheme,

$$
\begin{aligned}
\left.D_{n}^{(\rho)}\right|_{A} & =\left|\lambda_{2}\right|_{n}=\left|\hat{V}_{n}\right|=\left|C_{1} \hat{V}_{n_{1}}+C_{2} \hat{V}_{n_{2}}\right| \\
& \leq C_{1}\left|\hat{V}_{n_{1}}\right|+C_{2}\left|\hat{V}_{n_{2}}\right|=C_{1}\left|\lambda_{2}\right|_{n_{1}}+C_{2}\left|\lambda_{2}\right|_{n_{2}} \\
& =C_{1} D_{n_{1}}^{(\rho)}+C_{2} D_{n_{2}}^{(\rho)}=\left.D_{n}^{(\rho)}\right|_{R},
\end{aligned}
$$

where the subscript $A$ denotes the grid-Aligned scheme, and the subscript $R$ denotes the Rotated scheme. Obviously, $\left.D_{n}^{(\rho)}\right|_{A}$ is different from $\left.D_{n}^{(\rho)}\right|_{R}$, and the criterion Eq.26

not fulfilled in the rotated Roe scheme. Therefore, the rotated Roe scheme could not show exact solutions for contact discontinuity. Furthermore, based on Eq.27, the difference between the grid-aligned Roe scheme and the rotated Roe scheme could vary with the rotation angle, even if the local velocity is not changed.

For the rotated $\mathrm{AUSM}^{+}$scheme, similar to Eq.27, the dissipative density term shows following relation,

$$
\begin{aligned}
\left.D_{n}^{(\rho)}\right|_{A} & =\left|V_{1 / 2}\right|_{n}=\left|C_{1}\left(V_{1 / 2}\right)_{n_{1}}+C_{2}\left(V_{1 / 2}\right)_{n_{2}}\right| \\
& \leq C_{1}\left|V_{1 / 2}\right|_{n_{1}}+C_{2}\left|V_{1 / 2}\right|_{n_{2}} \\
& =C_{1} D_{n_{1}}^{(\rho)}+C_{2} D_{n_{2}}^{(\rho)}=\left.D_{n}^{(\rho)}\right|_{R} .
\end{aligned}
$$


Therefore, the rotation effects of the rotated $\mathrm{AUSM}^{+}$scheme are expected to be similar to those of the rotated Roe scheme.

For the rotated Van Leer scheme, we simplify the formula by assuming the problem is in subsonic flow condition. Therefore,

$$
D^{(\rho)}=\frac{1}{4}\left[a_{L}\left(M_{L}+1\right)^{2}+a_{R}\left(M_{R}-1\right)^{2}\right]
$$

For a stationary contact discontinuity in which $u_{c}=0$, both the grid-aligned and rotated Van Leer schemes are showing $D^{(\rho)} \geq 0$. In a rotation framework, the speed of sound remains the value of the grid-aligned calculation. It is easily deduced that

$$
C_{1}\left(M_{L, R}+1\right)_{n_{1}}^{2}+C_{2}\left(M_{L, R}+1\right)_{n_{2}}^{2} \geq\left(M_{L, R}+1\right)_{n}^{2}
$$

Therefore, $\left.D_{n}^{(\rho)}\right|_{R}$ will be larger than $\left.D_{n}^{(\rho)}\right|_{A}$. It is expected that the rotated Van Leer scheme is more dissipative than the grid-aligned Van Leer scheme.

The dissipative pressure terms of $\mathrm{AUSM}^{+}$scheme and Van Leer scheme are always equal to zero in both the grid-aligned and rotated frameworks. On the contrary, the term $D^{(p)}$ is not equal to zero in both the grid-aligned and rotated Roe scheme. Based on the conjecture of Liou, $D^{(p)} \neq 0$ is the root for causing the shock instabilities [5]. Although this conjecture has been challenged, to eliminate the $D^{(p)}$ term was regarded as an important part for improving the shock stability [22, 28, 4]. In the following sections, the actual performance of each upwind scheme will be examined by a serial of numerical cases.

\section{Numerical cases and discussions}

The numerical cases are designed to test the performance of rotated upwind schemes in both the strong shock stability and contact resolving capability. Therefore, numerical simulations of flat plate laminar boundary layer flow, subsonic viscous flow around a cylinder and inviscid hypersonic flow around a cylinder are conducted.

\subsection{Case 1: Flat plate laminar boundary layer}

The simulation of laminar boundary layer flow over a flat plat is usually used as a standard test for the accuracy of viscous flux formulations. Here, grid-aligned and rotated upwind schemes are investigated by a serial of simulations. 
The simulations are performed by Roe scheme, $\mathrm{AUSM}^{+}$scheme, Van Leer scheme and their rotated versions. Free stream Mach number is 0.3, Reynolds number is $10^{6}$. In order to reveal the effects of the rotation on upwind differencing direction, the results of rotated upwind schemes in three predefined rotation angles $\alpha=4.5^{\circ}, 22.5^{\circ}, 45^{\circ}$ are compared with those of the grid-aligned schemes and the Blasius' result.

The flow field is discretized into a quadrilateral grid, the height of the first layer of cells at the non-slip wall is 0.0001 , the aspect ratio of the grid cell that in the front of the flat plate is 1 . All the computations use LU-SGS scheme for temporal integration, the CFL number of which is $10^{6}$. Computations are deemed to be converged by 10 orders of magnitude reduction in the density residual. Velocity profiles across the boundary layer are compared. In order to present a common standard of diffusive results, the result of the grid-aligned Van Leer scheme is also presented along with the results of Roe and $\mathrm{AUSM}^{+}$schemes.

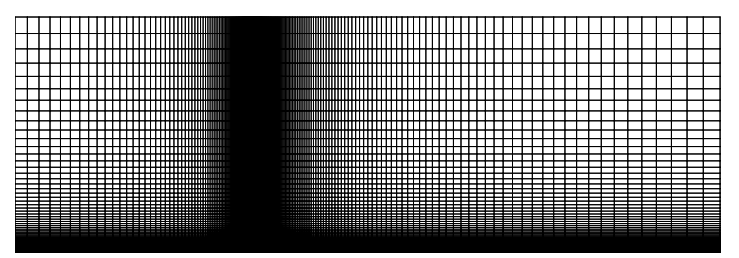

Figure 1: Grid for laminar boundary layer flow simulations

In Fig.2, the Roe scheme is investigated. Obviously, the grid-aligned Roe scheme shows accurate result, but the rotated Roe schemes present dissipative results. Thereinto, the result of the rotated Roe $\left(\alpha=22.5^{\circ}\right)$ scheme is similar to that of the Van Leer scheme, and the rotated Roe $\left(\alpha=45^{\circ}\right)$ scheme is more dissipative than the Van Leer scheme. Therefore, the rotation angles of the rotated Roe schemes significantly affects the contact resolution, and the dissipation will be more significant if the rotation angle becomes larger.

The $\mathrm{AUSM}^{+}$scheme presents accurate velocity profile, which is similar to the result of Roe scheme. However, dissipative results are presented by the rotated AUSM ${ }^{+}$ schemes. According to these results, rotation angle is an important factor for the accu- 


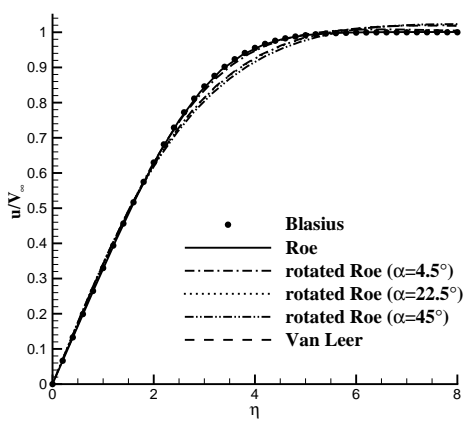

(a)

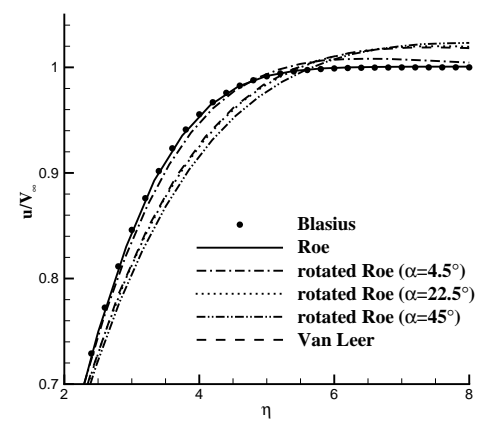

(b)

Figure 2: Velocity profile of laminar boundary layer flow: Roe and rotated Roe schemes

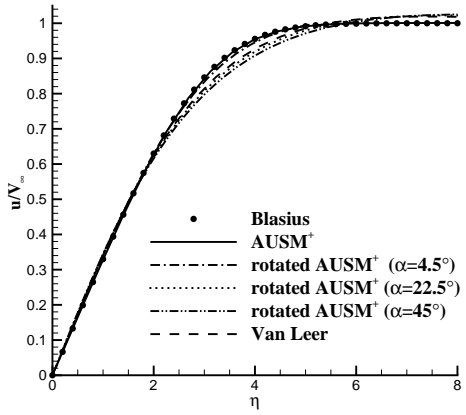

(a)

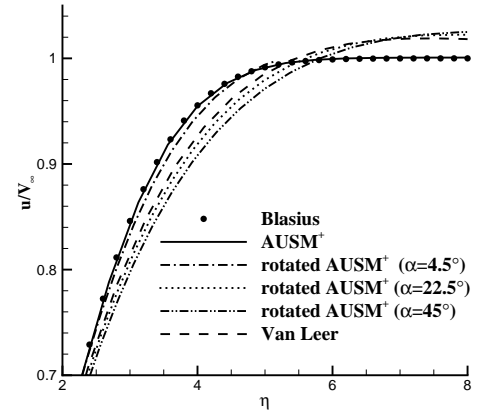

(b)

Figure 3: Velocity profile of laminar boundary layer flow: $\mathrm{AUSM}^{+}$and rotated $\mathrm{AUSM}^{+}$scheme

racy of boundary layer resolution. It should be noted that the rotated $\mathrm{AUSM}^{+}$schemes are more dissipative compared with the rotated Roe schemes.

In Fig.4, the grid-aligned Van Leer scheme shows inaccurate result, which is already shown in Fig.2 and Fig.3. Furthermore, the rotated Van Leer schemes are more dissipative compared with the grid-aligned scheme. This is supporting the analyses in subsection 4.2 .

In general, the boundary layer resolution of upwind schemes will be deteriorated 


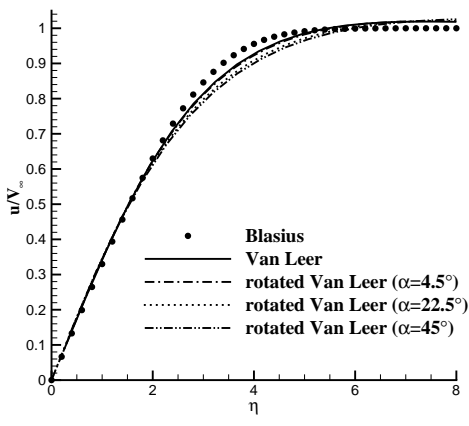

(a)

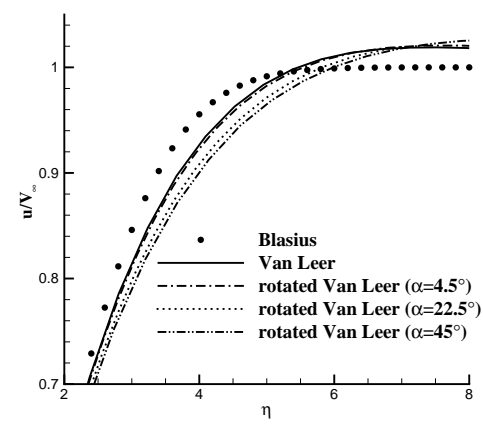

(b)

Figure 4: Velocity profile of laminar boundary layer flow: Van Leer and rotated Van Leer schemes

by the rotation on upwind differencing directions. The estimations for the velocity of contact discontinuity are inaccurate, as introduced in section 4 , and thus the deteriorated accuracy of the rotated upwind schemes could be easily predicted. However, the monotonicity of the dissipation with rotation angle, as has been shown in this section, will be a basic standpoint in this paper.

\subsection{Case 2: Subsonic viscous flow around a cylinder}

In the previous case, the rotation angles of the upwind differencing direction are predefined without consideration on local flow directions. In flat plate boundary layer flow, the streamlines are mostly conformed with grid lines and the flow gradients are approximately changing in one direction. Therefore, it is necessary to investigate the rotated upwind schemes in a more complicate flow field. Furthermore, the high aspectratio grid, which is used for the simulations of the dominant flow features in the former cases, might causes complicate numerical viscous effects [29]. In order to thoroughly investigate the dissipative effects of the rotated upwind schemes, two-dimensional subsonic flow around a cylinder [30] is simulated. In this case, the flow features are more complicate and the most part of the flow field is discretized by isotropic cells.

The simulations are performed on an O-type quadrilateral grid, as shown in Fig.5. The free stream Mach number is 0.3 and Reynolds number is 40 . The diameter of the 
cylinder is 1 , the height of first layer of cells is 0.001 . All the computations use the LU-SGS scheme of which the maximum CFL is 100, and the density residuals of the computations are reducing 10 order of magnitudes. Three definitions of upwind differencing directions are applied in this case. The first one is using 0 degree rotation angle, which leads to the grid-aligned scheme. The second one is the 45 degree angle rotated scheme, which is denoted as the MR (Maximum Rotation) scheme for simplicity in the following paragraphs. In the last definition, the directions for the convective fluxes computations are exactly accordance of the coordinate directions of two-dimensional plane, thus the scheme is isolated with grid geometry. The last scheme is denoted as CA (Coordinate Aligned) scheme.

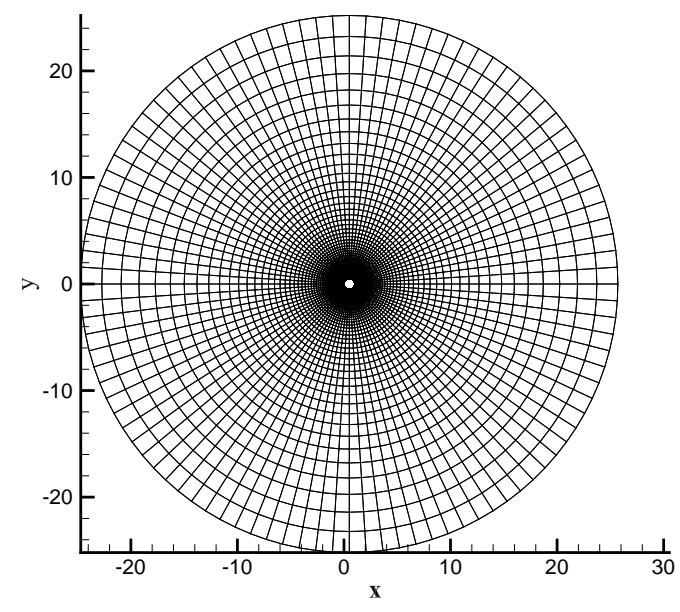

Figure 5: Grid for the simulation of subsonic flow around a cylinder

For brevity, only the streamlines in the results of the grid-aligned and rotated Roe schemes are visually shown in Fig.6. The lengths of the calculated closed wakes are presented in Table 1. Here, the exactness of the calculated wakes will be used as the estimation of the simulation accuracy. In general, the MR schemes present the smallest separated wake, which means the dissipation is significant.

The grid-aligned Roe scheme shows accurate result in the simulation. Obviously, 


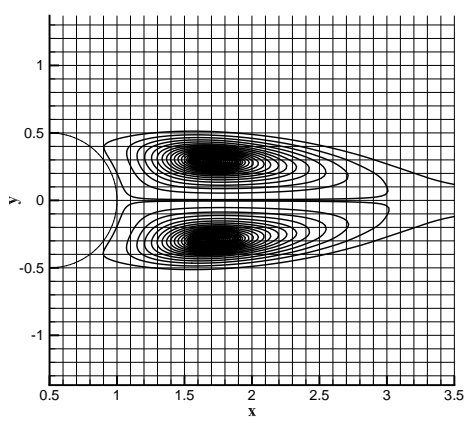

(a)

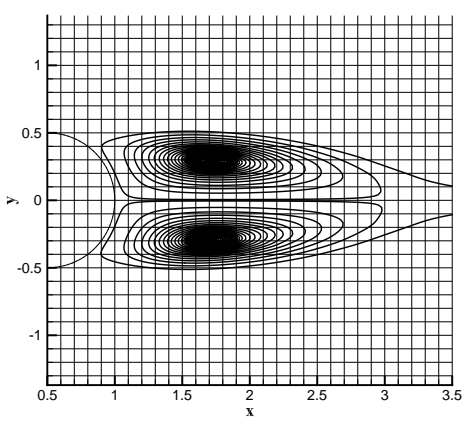

(b)

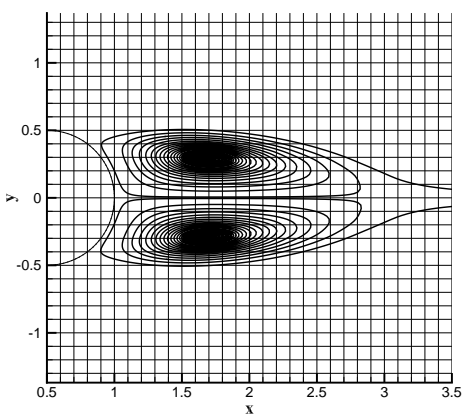

(c)

Figure 6: Streamlines of subsonic flow around cylinder. (a) Roe E-fix. (b) Roe CA. (c) Roe MR.

two rotated Roe schemes show poor accuracy. Especially, the Roe MR scheme shows tendencies of the dissipation of the rotated $\mathrm{AUSM}^{+}$schemes and rotated Van Leer schemes are the same as that of the rotated Roe schemes. It could be found that, the more significant rotation apply, the more dissipative result will show. Therefore, the monotonicity of the dissipation of rotated schemes with rotation angle is also held 225 in multidimensional cases. Rotation procedure on upwind schemes will substantially deteriorate the resolution of contact discontinuity and eventually disperse the viscous flow features. 
Table 1: Lengths of the closed wakes

\begin{tabular}{lcccc}
\hline & & Grid-Aligned & Coordinate-Aligned & Maximum Rotation \\
\hline Experiment & 2.13 & - & - & - \\
Roe & - & 2.135 & 2.095 & 1.925 \\
AUSM $^{+}$ & - & 2.097 & 2.044 & 1.849 \\
Van Leer & - & 1.922 & 1.869 & 1.659 \\
Roe WR-M & 2.132 & - & - & - \\
\hline
\end{tabular}

\subsection{Case 3: Inviscid hypersonic flow around a cylinder}

Last two cases show the behaviours of the rotated upwind schemes in the sim-

Firstly, the computation results of the grid-aligned schemes on quadrilateral grid are presented in Fig.8. A typical carbuncle phenomenon can be found in the result of Roe scheme. In fact, the Roe scheme could be showing shock instability in a Mach 


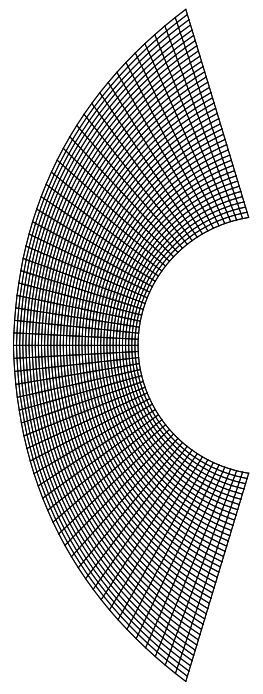

(a) Quadrilateral grid.

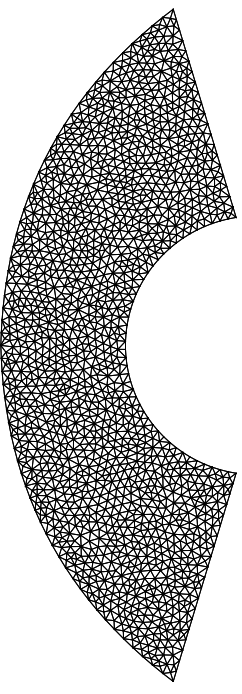

(b) Triangular grid.

Figure 7: Grids for simulations of inviscid hypersonic flow around a cylinder (boundary nodes are halved in the figures for clarity). lines. Slight anomalies are found in the result of $\mathrm{AUSM}^{+}$scheme. A zigzag-shaped shock wave that similar to the result of Shima et al [32] and folded contour lines in the stagnation point are shown. These results (or similar results) are already given by other researches. Secondly, Fig.9 presents the results that computed on the triangular grid. Obviously, $\mathrm{AUSM}^{+}$scheme shows carbuncle phenomenon as well as Roe scheme, but Van Leer scheme presents carbuncle-free result (even not as perfect as the result on quadrilateral grid). The result of $\mathrm{AUSM}^{+}$scheme further support the conclusion that $\mathrm{AUSM}^{+}$scheme could be denoted as a "Light carbuncle prone scheme" [6]. Finally, the results of the MR schemes are presented in Fig.10. Roe MR scheme, AUSM ${ }^{+}$MR scheme and Van Leer MR scheme are all showing carbuncle-free results.

Liou [5] suggested that the non zero dissipative pressure term should be the root of shock instabilities. Therefore, the grid-aligned $\mathrm{AUSM}^{+}$scheme and Van Leer scheme should be showing carbuncle-free results in the simulations. However, the AUSM ${ }^{+}$ scheme, which shows accurate boundary-layer resolution, is unstable in the simulation 


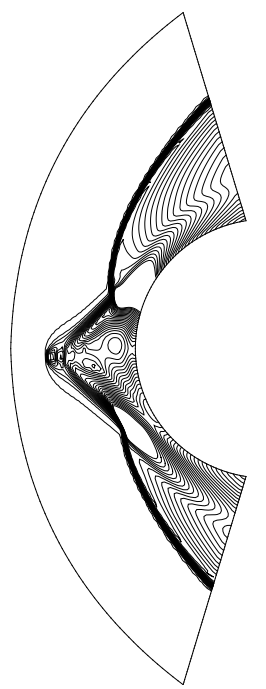

(a) Roe E-fix

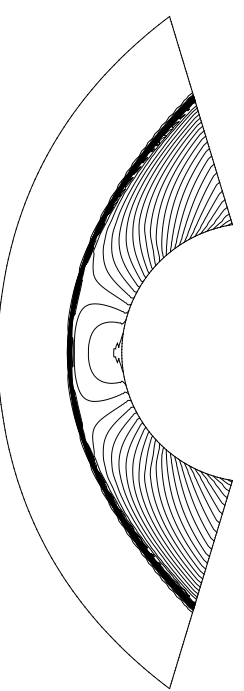

(b) $\mathrm{AUSM}^{+}$

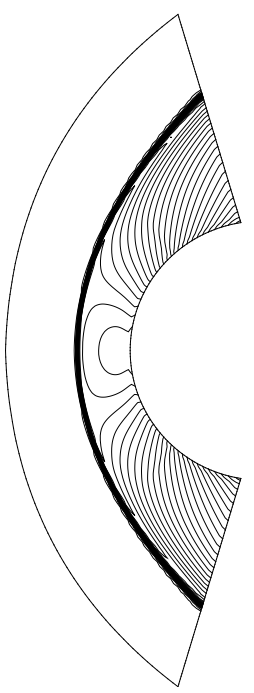

(c) Van Leer

Figure 8: Inviscid hypersonic flow around a cylinder at $M=20$ (grid-aligned solvers on quadrilateral grid). Thirty equally spaced contour lines from $\rho=1$ to $\rho=8$.

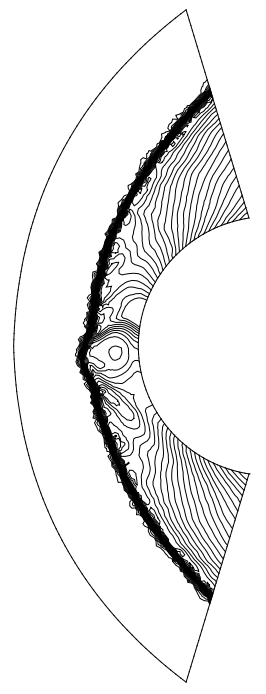

(a) Roe E-fix

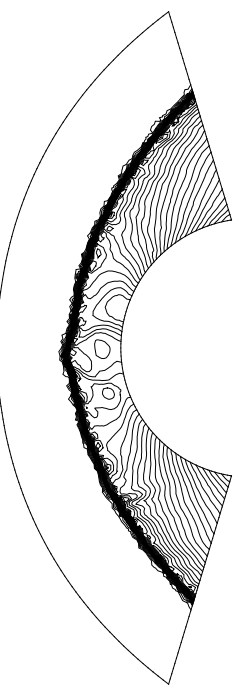

(b) $\mathrm{AUSM}^{+}$

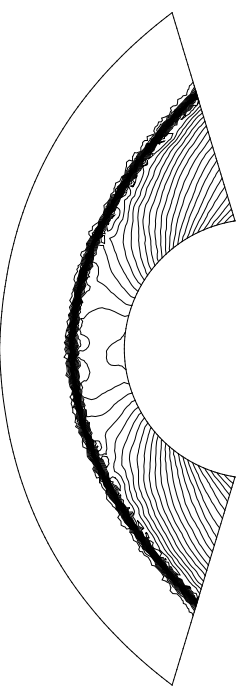

(c) Van Leer

Figure 9: Inviscid hypersonic flow around a cylinder at $M=20$ (grid-aligned solvers on triangular grid). Thirty equally spaced contour lines from $\rho=1$ to $\rho=8$. 


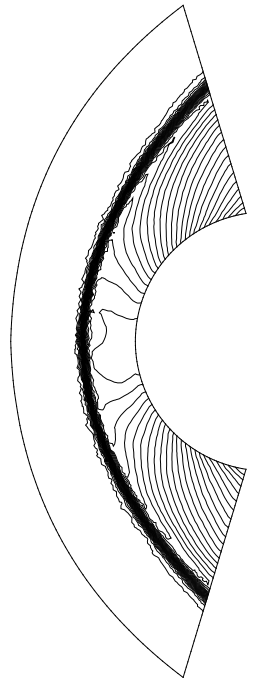

(a) Roe MR

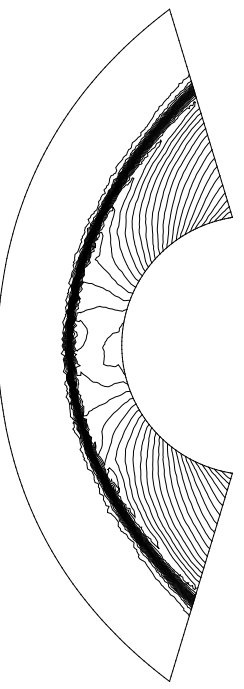

(b) $\mathrm{AUSM}^{+} \mathrm{MR}$

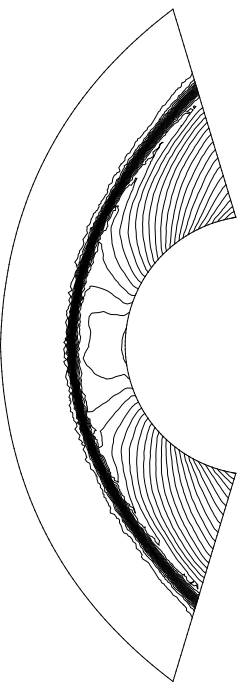

(c) Van Leer MR

Figure 10: Inviscid hypersonic flow around a cylinder at $M=20$ (rotated solvers on triangular grid). Thirty equally spaced contour lines from $\rho=1$ to $\rho=8$.

265

on the triangular grid. On the other hand, all the schemes of which the dissipative density term does not fulfill Eq.26 are showing carbuncle-free results even on the triangular grid. In particular, the Roe MR scheme, in which $D^{(p)} \neq 0$, is also showing a shock stable result. It is not suggesting that the pressure terms in the numerical mass fluxes are not affecting the shock stabilities. It is more likely that the perturbations introduced of rotated upwind schemes, as the similar result of the grid-aligned Roe scheme [22].

\section{A pressure-weighted rotation strategy}

For the rotated upwind schemes, the previous paragraphs are suggesting that the extra dissipation of the density term in numerical mass fluxes will dump the shock instabilities. Meanwhile, the rotated upwind schemes are showing deteriorated accuracy in the simulations of viscous flows. Because the monotonicity of the dissipation with rotation angle is shown, it will be possible to find a strategy to improve the performance 
of rotated upwind schemes. A straightforward strategy is to control the rotation angle by the shock strength. Here, the rotated Roe scheme is redefined by using the strategy.

\subsection{Roe WR-M scheme}

The key of the proposed strategy is tuning the dissipation by local shock strength, therefore a shock detection method is requested. The hybrid FVS scheme of Drikakis and Tsangaris [10] used the eigenvalues of numerical split fluxes as indicators for the activation of the second-order dissipation terms. Quirk [2] presented a pressurebased switching function for the adaptive application of HLLE scheme [33]. Here, the pressure-weight function of Kim et al [34] is used as a basic indicator of shock strength. The function is

$$
f=1-\min \left(\frac{p_{L}}{p_{R}}, \frac{p_{R}}{p_{L}}\right)^{3} .
$$

This function value is gradual changing by the pressure difference at two sides of a interface, and the function has proved to be stable for switching flux functions.

When the numerical flux across a certain cell interface needs to be calculated, as shown in Fig.11, the shock-detective function for the hybridization of fluxes should examine all the adjacent interfaces [22]. Here, the function is defined for selecting the maximum $f_{k}$ value at the interfaces of both cell $i$ and $j$ :

$$
\omega=\max _{k}\left(f_{k}\right)
$$

In regions of smooth flows, $f_{k}$ of each interface will be in a similar magnitude, and thus the maxima function will not significantly affect calculation results.

For simplicity, the left or right pressure in function $f$ is directly using the left or right cell center value, in both first-order and second-order spatial accuracy applications. In the regions of strong shocks, the difference between left and right pressure is large, and thus the function value $\omega$ will be approximately equal to 1 . In regions of smooth flows, the function value will be very small. Therefore, the function $\omega$ will be suitable for indicating the existence of shock waves.

The rotation angle is then defined as

$$
\alpha=\frac{\pi}{4} * \omega .
$$




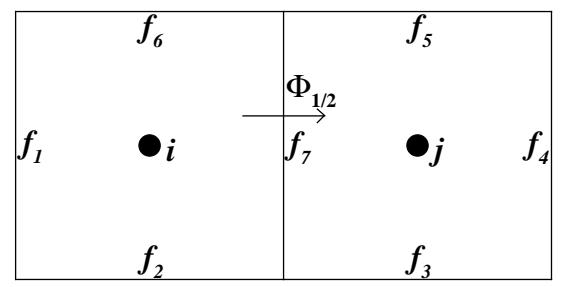

Figure 11: Illustration of referenced pressure weight functions, $f_{k}$, of the present shock-detective function, $\omega$, for the calculation of an unknown flux, $\Phi_{1 / 2}$.

Ultimately, the rotation angle is determined by the strength of the local pressure difference. In the vicinity of a strong shock, the angle $\alpha$ will be approximately equal to 45 degree. Conversely, the grid-aligned or "nearly" grid-aligned scheme, in which the $\alpha$ is approximately equal to 0 degree, will be used in the region without strong pressure discontinuity. Therefore, according to the previous analyses, the extra dissipation of the rotated Roe scheme will be introduced only for the calculation of shock waves. Here, the presented rotated Roe scheme is named as Roe WR-M (Weighted Rotation-Multidimensional) scheme for brevity.

\subsection{Notes on three-dimensional cases}

In three-dimensional cases, the unit direction vector $\mathbf{n}_{3}$ which obeys conditions $\mathbf{n}_{1} \cdot \mathbf{n}_{3}=0, \mathbf{n}_{2} \cdot \mathbf{n}_{3}=0$ and $C_{3}=\mathbf{n}_{3} \cdot \mathbf{n}$, needs to be introduced. In the rotated schemes of Ren or Nishikawa, $\mathbf{n}_{3}$ could be defined by a simple cross product of $\mathbf{n}_{1}$ and $\mathbf{n}_{2}$, and thus $C_{3}$ is equal to zero. Correspondingly, the $\mathbf{n}_{3}$ is not directly contributing to the calculation of numerical fluxes.

The newly proposed definition of directions $\mathbf{n}_{1}$ and $\mathbf{n}_{2}$ is given by Eq.13. In threedimensional cases, the $\mathbf{n}_{3}$ should be defined specifically. Here, the local flow velocity direction is introduced as $\mathbf{n}_{V} \|\left(\mathbf{V}_{L}+\mathbf{V}_{R}\right)$, at each interface, and then $\mathbf{n}_{3}$ will be defined to be parallel to $\mathbf{n}_{V} \times \mathbf{n}$. In other words, the rotation of two directions, $\mathbf{n}_{1}$ and $\mathbf{n}_{2}$, will be performed in a plane constructed by the flow velocity direction $\mathbf{n}_{V}$ and face normal direction $\mathbf{n}$. In fact, the inconsistent normal momentum distribution along shock front, which was suggested to be the source of shock instabilities [35], could be dumped by 
the dissipative term that is produced in the rotation plane of each interface. This is because the dissipative term that smear the contact surface could also dump the tangent velocity discontinuity, and then the momentum perturbation could be suppressed. Simcompleteness, a three-dimensional numerical cases is presented in the next subsection.

\subsection{Numerical results}

By using the proposed rotation strategy, more dissipation will be introduced into flux calculations. Therefore, numerical cases on viscous flow simulations are shown 335 at first, by which the accuracy of the rotated scheme will be clarified. Here, flat plate laminar boundary layer flow and subsonic flow around a cylinder are both calculated. In Fig.12(a), an accurate velocity profile of laminar boundary layer is presented. The separated wake behind the cylinder is shown in Fig.12(b), and the length of the closed wake is presented in Table 1. The length of the calculated closed wake is close to the numerical result of the grid-aligned Roe scheme and the experiment result. These results are produced due to the rotated scheme Roe WR-M is acting as the grid-aligned 


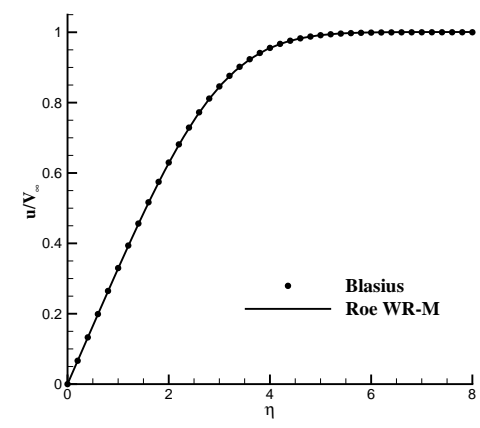

(a) Velocity profile of laminar boundary layer

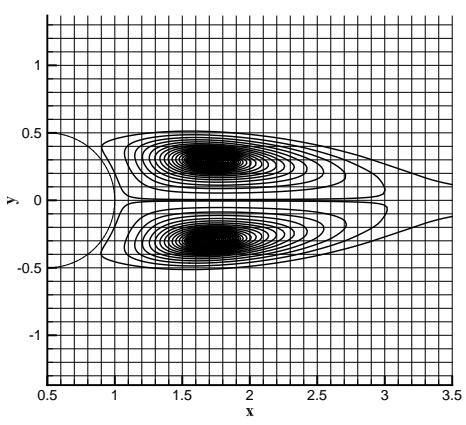

(b) Streamline of subsonic flow around cylinder

Figure 12: Viscous flow simulation results of Roe WR-M scheme.

Roe scheme in the boundary layer and wake region, which are showing small pressuregradient.

The simulation of inviscid hypersonic flow around a cylinder is made by the proposed rotated scheme. As the former work on upwind-differencing schemes [36], in order to investigate the effects of Mach number, numerical simulations of a wide range Mach number are performed (the other conditions are the same as in subsection 5.3). Part of the results are shown in Fig.13. The Roe WR-M scheme is showing carbunclefree results in all the cases. Whereas, the Roe scheme is failed to produce shock stable results in these cases. This is a evidence that the extra dissipation nearby the shock wave has dumped the shock instability. In these cases, the shock-detective function introduces the extra dissipation based the pressure-difference at each interface, and shows adaptability on the variation of Mach number.

The double-Mach reflection (DMR) problem is simulated for showing the performance on unsteady problem. This problem was studied extensively by Woodward and Colella [37]. Initially, a Mach 10 shock wave is located at $x=0$, and then the moving shock travels in the x-direction and encounters a wedge that makes an angle $30^{\circ}$ with the shock propagation (x-direction). The air ahead of the shock wave has a state $(\rho, u, v, p)=(1.4,0,0,1)$. The computational domain is $[-0.5,2.5] \times[0,2.5]$ and a $300 \times 300$ quadrilateral grid is used for discretization. The computations use the 


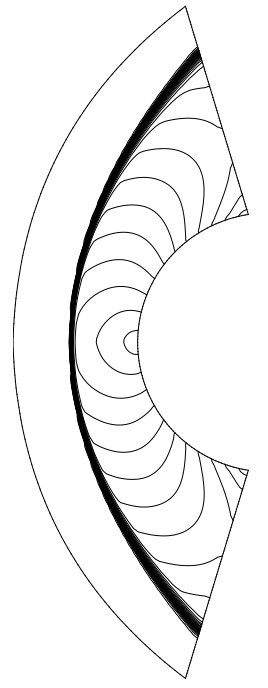

(a) Mach 5

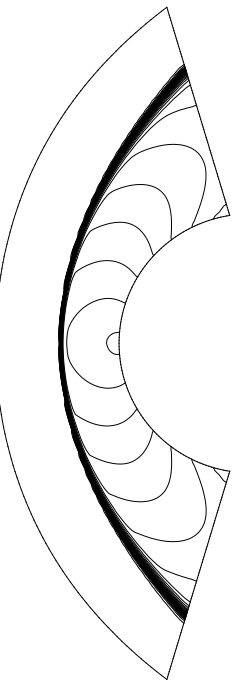

(b) Mach 8

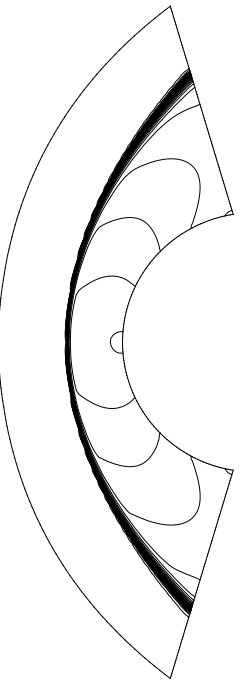

(c) Mach 12.8

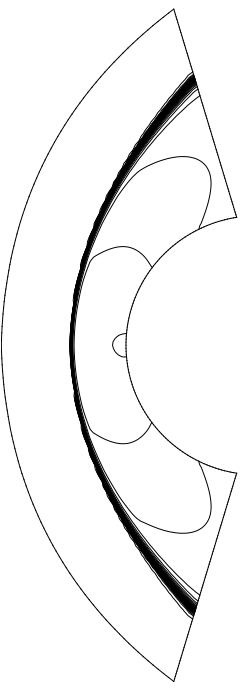

(d) Mach 20

Figure 13: Mach contours of inviscid hypersonic flow around a cylinder (Roe WR-M on quadrilateral grid). Thirty equally spaced contour lines from minimum Mach number to maximum Mach number.

explicit Runge-Kutta scheme for temporal solution. The rotated scheme is also compatible with the implicit scheme as in [38]. The CFL is 1 and computations run up to the dimensionless time $t=0.2$. The second-order finite volume method is used for spatial discretization. The Venkatakrishnan's limiter, of which the parameter $K$ is set as 1 , is used for remaining the monotonicity in the vicinity of shock waves. The results are shown in Fig.14. For the Roe scheme the primary Mach stem is kinked. Whereas, the rotated scheme Roe WR-M is showing shock stability.

As shown in Fig.15, a three-dimensional case is also used to examine the Roe WR-M scheme. The $120 \times 80 \times 25$ grid for the simulation of hypersonic flow around a cylinder is made by extruding the two-dimensional quadrilateral grid along the zdirection. The other calculation conditions remain the same as the two-dimensional case of which the Mach number is 20 . The rotated scheme is showing carbuncle-free result in this case. Whereas, the Roe scheme shows significant carbuncle phenomenon. 


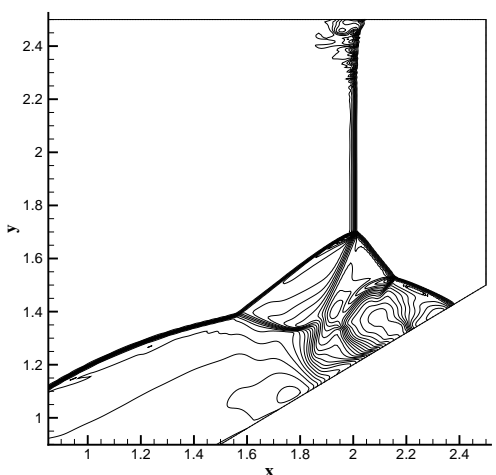

(a) Roe E-fix

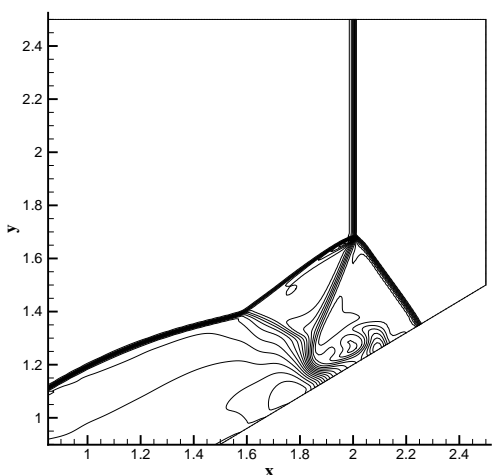

(b) Roe WR-M

Figure 14: The density contours of the double Mach reflection problem. Thirty equally spaced contour lines from $\rho=4$ to $\rho=24$.

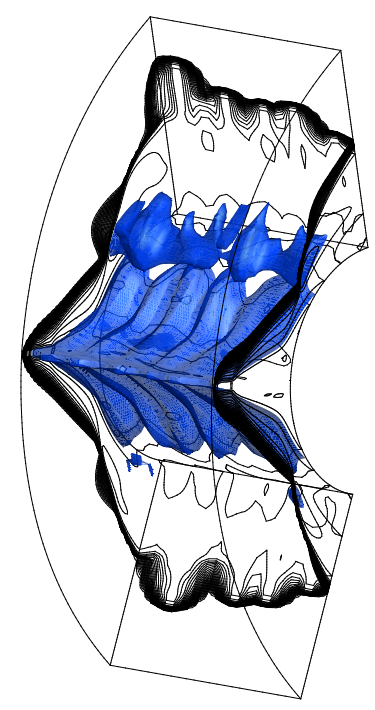

(a)

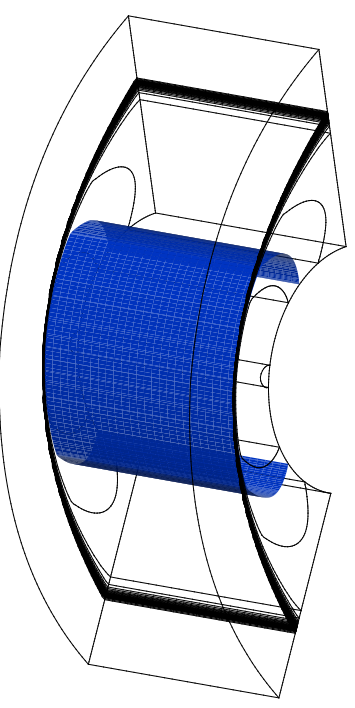

(b)

Figure 15: a) Snapshots of developing 3-D carbuncle of Roe E-Fix. b) A carbuncle-free result of Roe WR-M. Thirty equally spaced Mach contour lines from 0.0 Mach to 20.0 Mach and iso-Mach-number surface of 1.0 Mach. 


\subsection{Notes on the tunable dissipation}

375 tuned by the presented rotation strategy, and shock stability and contact resolved capability could be acquired at the same time. It should be noted that the application of tunable dissipation for improving shock stability is not new. The hybrid FVS scheme of Drikakis and Tsangaris [10] was directly applying a second-order artificial dissipaschemes. On the other hand, the hybrid scheme that combines upwind fluxes [2] was introducing the tunable dissipation by adaptively applying the dissipative flux function. This strategy has leaded to a serial of subsequent works. In this paper, comparisons to other hybrid schemes are not presented due to the depth of the scope. However, the proposed rotated scheme shows a different way of exploiting the dissipation mechanism of rotated schemes.

\section{Conclusions}

In this work, the shock stability and contact resolving capability of rotated upwind schemes are investigated by theoretical and numerical investigations. Three typical and commonly used upwind schemes, including Roe scheme, $\mathrm{AUSM}^{+}$scheme, Van Leer scheme, and their rotated versions are discussed.

Based on the former researches, theoretical analyses are conducted by investigating the dissipative terms in the numerical mass fluxes of upwind schemes. The dissipative terms of three grid-aligned upwind schemes are briefly introduced and then the rotated upwind schemes are analysed. In general, the rotation procedure will change the dissipative terms. Most importantly, the dissipative density term, which is critical for the exact solution of contact discontinuity, will be changed. Therefore, the accuracy of rotated upwind schemes will be deteriorated. On the other hand, the dissipative pressure term is not equal to zero in both the rotated Roe scheme and grid-aligned Roe scheme.

procedure. 
In the numerical cases, it is confirmed that the accuracy of the simulations for viscous flow will be deteriorated by the rotation on the upwind differencing direction. Thereinto, the rotated upwind schemes show inaccurate results in the simulations of flat plate boundary layer flow and subsonic flow around a cylinder. Furthermore, the monotonicity of the dissipation of the rotated schemes with their rotation angle is found in the simulations. Shock stabilities of the rotated schemes are also tested. The rotated schemes show shock stable results on both the quadrilateral grid and triangular grid. A pressure-weighted rotation strategy is then introduced, and showing both shock stability and accurate resolution for contact discontinuity. Different from the former researches that concentrate on a physical meaningful upwind direction, the tuneable dissipation of the presented strategy is determined mainly by the dissipative terms in the numerical mass flux, and could be controlled by the rotation angle of upwind direction. Numerical results prove that the dissipation of the proposed rotation strategy could be effective for suppressing the momentum perturbation which might causes multidimensional shock instabilities.

Further conclusions can be made based on above direct observations. As mentioned in former researches, perturbations could be evolved by the pressure term in numerical mass flux, thus the shock stabilities of upwind schemes are related to the dissipative pressure term. However, the condition $D^{(p)}=0$ is not sufficient for a scheme to prevent the shock instability from occurring, especially while the simulations are performed on unstructured grid. On the other hand, the perturbations can be effectively dumped by a proper dissipative density term. This is why the rotated upwind schemes are showing intrinsic dissipative property. The intrinsic dissipation, which is essentially introduced by the inaccurate estimation on the velocity of contact discontinuity, will deteriorate the resolution of contact discontinuities and improve the shock stabilities. Therefore, the performance of upwind schemes can be improved by adaptively tuning the dissipation.

\section{Acknowledgments}

This work was supported by the National Natural Science Foundation of China under Grant 91315302 and 11372064; 111 Project under Grant B14013; and Funda- 
mental Research Funds for the Central Universities under Grant (DUT15JJ(G)03). We also thank the anonymous reviewers for their constructive comments and suggestions.

\section{References}

[1] K. Peery, S. Imlay, Blunt-body flow simulations, 24th Joint Propulsion Conference, AIAA Paper 88-2904, Bellevue, WA, 1988. doi : 10. 2514/6.1988-2904.

[2] J. J. Quirk, A contribution to the great Riemann solver debate, Int. J. Numer. Meth. Fluids 18 (6) (1994) 555-574.

[3] K. Kitamura, P. Roe, F. Ismail, Evaluation of Euler fluxes for hypersonic flow computations, AIAA Journal 47 (1) (2009) 44-53. doi : 10.2514/1 .33735.

[4] K. Kitamura, E. Shima, P. L. Roe, Carbuncle phenomena and other shock anomalies in three dimensions, AIAA Journal 50 (12) (2012) 2655-2669. doi:10.2514/1. J051227.

[5] M.-S. Liou, Mass flux schemes and connection to shock instability, Journal of Computational Physics 160 (2) (2000) 623-648.

[6] M. Pandolfi, D. D'Ambrosio, Numerical instabilities in upwind methods: Analysis and cures for the "Carbuncle" phenomenon, Journal of Computational Physics 166 (2) (2001) 271-301.

[7] J. Gressier, J.-M. Moschetta, Robustness versus accuracy in shock-wave computations, Int. J. Numer. Meth. Fluids 33 (3) (2000) 313-332.

[8] A. Eberle, M. A. Schmatz, N. C. Bissinger, Generalized fluxvectors for hypersonic shock-capturing, 28th Aerospace Sciences Meeting, AIAA Paper 90-0390, Reno, NV, 1990.

[9] D. Drikakis, S. Tsangaris, On the solution of the compressible navier- stokes equations using improved flux vector splitting methods, Applied Mathematical Modelling 17 (6) (1993) 282-297. 
[10] D. Drikakis, S. Tsangaris, On the accuracy and efficiency of CFD methods in real gas hypersonics, Int. J. Numer. Meth. Fluids 16 (9) (1993) 759-775.

[11] J. Zółtak, D. Drikakis, Hybrid upwind methods for the simulation of unsteady shock-wave diffraction over a cylinder, Computer Methods in Applied Mechanics and Engineering 162 (1-4) (1998) 165-185.

[12] D. W. Levy, K. G. Powell, B. van Leer, Use of a rotated Riemann solver for the two-dimensional Euler equations, Journal of Computational Physics 106 (2) (1993) 201-214.

[13] Y. X. Ren, A robust shock-capturing scheme based on rotated Riemann solvers, Computers \& Fluids 32 (10) (2003) 1379-1403.

[14] P. L. Roe, Approximate Riemann solvers, parameter vectors, and difference schemes, Journal of Computational Physics 43 (2) (1981) 357-372.

[15] H. Nishikawa, K. Kitamura, Very simple, carbuncle-free, boundary-layerresolving, rotated-hybrid Riemann solvers, Journal of Computational Physics 227 (4) (2008) 2560-2581.

[16] A. Harten, P. D. Lax, B. van Leer, On upstream differencing and Godunov-type schemes for hyperbolic conservation laws, SIAM Review 25 (1) (1983) 35-61.

[17] G. Zha, Y. Shen, M. Huerta, Rotated hybrid low diffusion ecusp-hll scheme and its applications to hypersonic flows, 20th AIAA Computational Fluid Dynamics Conference, AIAA Paper 2011-3545, Honolulu, HI, 2011. doi:10.2514/6.2011-3545.

[18] A. Jameson, Analysis and design of numerical schemes for gas dynamics, 1: Artificial diffusion, upwind biasing, limiters and their effect on accuracy and multigrid convergence, International Journal of Computational Fluid Dynamics 4 (3-4) (1995) 171-218. doi:10.1080/10618569508904524.

[19] A. Jameson, Analysis and design of numerical schemes for gas dynamics, 2: Artificial diffusion and discrete shock structure, Interna- 
tional Journal of Computational Fluid Dynamics 5 (1-2) (1995) 1-38. doi:10.1080/10618569508940734.

485

[23] J. Blazek, Computational Fluid Dynamics: Principles and Applications, 2nd Edition, Elsevier, 2005.

[24] T. J. Barth, D. C. Jespersen, The design and application of upwind schemes on

[28] E. Shima, K. Kitamura, Parameter-free simple low-dissipation AUSMfamily scheme for all speeds, AIAA Journal 49 (8) (2011) 1693-1709. doi:10.2514/1. J050905. 
[29] K. Xu, Z. Li, Dissipative mechanism in godunov-type schemes, Int. J. Numer. Meth. Fluids 37 (1) (2001) 1-22.

[30] M. Coutanceau, R. Bouard, Experimental determination of the main features of the viscous flow in the wake of a circular cylinder in uniform translation. part 1. steady flow, Journal of Fluid Mechanics 79 (02) (1977) 231-256.

[31] A. Harten, High resolution schemes for hyperbolic conservation laws, Journal of Computational Physics 49 (3) (1983) 357-393.

[32] E. Shima, K. Kitamura, Multidimensional numerical noise from captured shock wave and its cure, AIAA Journal 51 (4) (2013) 992-998. doi:10.2514/1. J052046.

[33] B. Einfeldt, On Godunov-type methods for gas dynamics, SIAM Journal on Numerical Analysis 25 (2) (1988) 294-318.

[34] K. H. Kim, J. H. Lee, O. H. Rho, An improvement of AUSM schemes by introducing the pressure-based weight functions, Computers \& Fluids 27 (3) (1998) 311-346.

[35] Z. Shen, W. Yan, G. Yuan, A stability analysis of hybrid schemes to cure shock instability, Communications in Computational Physics 15 (5) (2014) 1320-1342.

[36] S. Tissera, D. Drikakis, T. Birch, Computational fluid dynamics methods for hypersonic flow around blunted-cone-cylinder-flare, Journal of Spacecraft and Rockets 47 (4) (2010) 563-570. doi:10.2514/1.46722.

[37] P. Woodward, P. Colella, The numerical simulation of two-dimensional fluid flow with strong shocks, Journal of Computational Physics 54 (1) (1984) 115-173.

[38] A. Bagabir, D. Drikakis, Numerical experiments using high-resolution schemes for unsteady, inviscid, compressible flows, Computer Methods in Applied Mechanics and Engineering 193 (42-44) (2004) 4675-4705. 